\title{
CUMULATIVE SHOCK MODELS
}

\author{
ALLAN GUT, ${ }^{*}$ Uppsala University
}

\begin{abstract}
We show that a theory for stopped two-dimensional random walks is well suited to describe cumulative shock models. Limit theorems for the lifetime/failure time of a system are provided.

SHOCK; INTERSHOCK TIME; STOPPED RANDOM WALK; RENEWAL THEORY; FIRST-PASSAGE TIMES; STRONG LAW; CENTRAL LIMIT THEOREM; LAW OF THE ITERATED LOGARITHM; MOMENT CONVERGENCE; INSURANCE RISK THEORY
\end{abstract}

\section{Introduction}

The aim of this note is to show that a theory for stopped two-dimensional random walks, introduced in Gut and Janson (1983) and further developed in Gut (1988), Chapter IV, is very well suited to describe cumulative shock models. We thereby generalize and extend results of Sumita and Shanthikumar (1985) and, also, provide simpler proofs.

The general setup in cumulative shock models is a family $\left\{\left(X_{k}, Y_{k}\right), k \geqq 0\right\}$ of i.i.d. two-dimensional random variables, where $X_{k}$ represents the magnitude of the $k$ th shock and where $Y_{k}$ represents the time between the $(k-1)$ th and the $k$ th shock in model I and the time between the $k$ th and the $(k+1)$ th shock in model II. The system breaks down when the cumulative shock magnitude exceeds some given level. The object in focus is the lifetime/failure time of the system.

In earlier work it is assumed that the individual summands are non-negative, something that is very reasonable for time and shocks, although one could certainly imagine a model where (at least) shocks may be both positive and negative. In the present setup we do not need the assumption of non-negativity; only that the random walk that is generated by the shocks has positive drift.

To make the note reasonably self-contained we present, in Section 2, a short background of the theory of stopped two-dimensional random walks mentioned above. In Section 3 we treat shock model I and in Section 4 shock model II. Finally, Section 5 contains an application to insurance risk theory.

\section{Preliminaries}

In this section we present the general model of Gut and Janson (1983); see also Gut (1988), Section IV.2.

Let $\left\{\left(W_{k}, Z_{k}\right), k \geqq 1\right\}$ be a sequence of i.i.d. two-dimensional random variables with $\mu_{w}=E W_{1}>0\left(W_{0}=Z_{0}=0\right)$. No assumptions are made about independence or dependence between $W_{k}$ and $Z_{k}$ for any $k$. Further, set $U_{n}=\sum_{k=1}^{n} W_{k}$ and $V_{n}=\sum_{k=1}^{n} Z_{k}, n \geqq 1$, and define the first-passage-time process $\{\tau(t), t \geqq 0\}$ by

$$
\tau(t)=\min \left\{n: U_{n}>t\right\} .
$$

The random variable of interest is $V_{\tau(t)}$, and in the work cited above limit laws for $V_{\tau(t)}$ as $t \rightarrow \infty$ are derived. We refer the reader to these sources for details.

Received 17 May 1989; revision received 4 December 1989.

* Postal address: Uppsala University, Department of Mathematics, Thunbergsvägen 3, S-752 38 Uppsala, Sweden. 


\section{The cumulative shock model I}

In model I $Y_{k}$ is the time between the $(k-1)$ th and the $k$ th shock and $\left\{\left(X_{k}, Y_{k}\right), k \geqq 1\right\}$ are i.i.d. random variables; $X_{0}=Y_{0}=0$.

By identifying $X_{k}$ with $W_{k}$ and $Y_{k}$ with $Z_{k}$ (of Section 2) for $k \geqq 1$, we note that $U_{n}$ represents the cumulative magnitude of the shocks caused by the first $n$ shocks, that $V_{n}$ represents the time that has elapsed at the time of the $n$th shock, that $\tau(t)$ equals the number of shocks that have occurred when the cumulative shock magnitude exceeds $t$ (units) and that $V_{\tau(t)}$ is the time that has elapsed at the moment of occurrence of the $\tau(t)$ th shock. In particular, if the system fails as soon as the cumulative shock magnitude exceeds the level $u$ it follows that for the lifetime of the system (the failure time of the system), $T(u)$, we have $T(u)=V_{\tau(u)}$.

We are thus in the position that every theorem in Gut (1988), Section IV.2 can be applied to the present model. As a sample we state the following result.

Theorem I. (i) If $\mu_{x}=E X_{1}>0$ and $\mu_{y}=E Y_{1}$ exist, finite, then

$$
\frac{T(u)}{u} \rightarrow \frac{\mu_{y}}{\mu_{x}} \text { a.s. and in } L^{1} \quad \text { as } \quad u \rightarrow \infty \text {. }
$$
then

(ii) If, in addition, $\sigma_{x}^{2}=\operatorname{Var} X_{1}$ and $\sigma_{y}^{2}=\operatorname{Var} Y_{1}$ are finite and $\gamma^{2}=\operatorname{Var}\left(\mu_{x} Y_{1}-\mu_{y} X_{1}\right)>0$,

$$
\frac{T(u)-\left(\mu_{y} / \mu_{x}\right) u}{\sqrt{\mu_{x}^{-3} \gamma^{2} u}} \rightarrow \mathrm{N}(0,1) \text { in distribution and in } L^{2} \text { as } u \rightarrow \infty .
$$

(iii) Let $C\left(\left\{x_{n}\right\}\right)$ denote the set of limit points of the sequence $\left\{x_{n}\right\}$. Under the assumptions of (ii) we have

in particular,

$$
C\left(\left\{\frac{T(u)-\left(\mu_{y} / \mu_{x}\right) u}{\sqrt{2 \mu_{x}^{-3} \gamma^{2} u \log \log u}}\right\}\right)=[-1,1] \text { a.s., }
$$

$$
\limsup _{u \rightarrow \infty}\left(\liminf _{u \rightarrow \infty}\right) \frac{T(u)-\left(\mu_{y} / \mu_{x}\right) u}{\sqrt{2 u \log \log u}}=( \pm) \frac{\gamma}{\mu_{x}^{3 / 2}} \quad \text { a.s. }
$$

\section{Remarks}

3.1. Recall that we do not assume that $\left\{X_{k}\right\}$ or $\left\{Y_{k}\right\}$ is non-negative; only that $E X_{k}>0$. Further, nothing is assumed concerning independence/dependence between $X_{k}$ or $Y_{k}, k \geqq 1$.

3.2. It follows, in particular, from (ii) that $E T(u)=\left(\mu_{y} / \mu_{x}\right) u+o(u)$ as $u \rightarrow \infty$. However, it is true that $\operatorname{ET}(u)=\left(\mu_{y} / \mu_{x}\right) u+$ constant $+o(1)$ as $u \rightarrow \infty$, where the constant depends on the first two moments of $X_{1}$ and $Y_{1}$, the ladder variables of the $X$-sequence and on whether or not the distribution of this sequence is arithmetic.

Further, if $\mu_{y}=0$, then $E T(u)=0$ and $\operatorname{Var} T(u)=\left(\sigma_{y}^{2} / \mu_{x}\right) u+o(u)$ as $u \rightarrow \infty$. (This is, of course, not possible if time is non-negative.)

3.3. Sumita and Shanthikumar (1985) assume that shocks and time are non-negative and that the distributions are absolutely continuous with finite third moments. Their proofs are based on transforms. Their proof of the representation formula for the failure time yields equality in distribution (see their Theorem 2.A3), a result which is too weak for a.s. statements. Their result on asymptotic normality (Theorem 2.A9) is proved under additional independence assumptions.

3.4. Anderson (1988) provides limit theorems for $T(u)$ in the case of attraction to stable laws with index $\leqq 1$.

3.5. Further results that can be obtained via Gut (1988), Section IV.2 are Marcinkiewicz laws, convergence to stable distributions with index between 1 and 2 and joint asymptotic normality of the lifetime and the number of shocks until failure. 


\section{The cumulative shock model II}

As before, $\left\{X_{k}, k \geqq 1\right\}$ are magnitudes of the shocks, $\left\{Y_{k}, k \geqq 1\right\}$ are the intershock times and $\left\{\left(X_{k}, Y_{k}\right), k \geqq 1\right\}$ are i.i.d. random variables. However, in this model $Y_{k}$ is the time between the $k$ th and the $(k+1)$ th shock. We therefore also need $Y_{0}$, the time until the occurrence of the first shock, which is such that $\left\{Y_{k}, k \geqq 0\right\}$ are i.i.d. random variables. Also, $X_{0}=0$.

The identification with Section 2 now becomes $X_{k} \leftrightarrow W_{k}$ for $k \geqq 1, Y_{k} \leftrightarrow Z_{k}$ for $k \geqq 0$; we thus do not have $Z_{0}=0$ in this case. With $\tau(t)$ as before the lifetime of the system, $T(u)$, now becomes

$$
T(u)=Y_{0}+V_{\tau(u)-1}\left(=\sum_{k=0}^{\tau(u)-1} Y_{k}\right)
$$

This model thus is, mathematically, more complicated than model I because

(a) $\tau(t)-1$ is not a stopping time;

(b) the distribution of $\left(X_{0}, Y_{0}\right)$ differs from that of $\left(X_{k}, Y_{k}\right), k \geqq 1$.

These problems can, however, be taken care of. For example, in order to prove asymptotic normality it is not necessary that the family of random indices is a family of stopping times; a weak law of large numbers suffices. That such a law holds follows from renewal theory (on the line). Further, the addition of $Y_{0}$ to the sum $V_{\tau(u)-1}$ clearly does not destroy the convergence and it follows that $T(u)$ is asymptotically normal as $u \rightarrow \infty$ also in the present context. The same is true, similarly, for the strong law and the law of the iterated logarithm.

As for uniform integrability and moment convergence it is essential that $\tau(t), t \geqq 0$, are stopping times. However, $Y_{\tau(u)}$ can be taken care of by Gut (1988), Theorem I.8.1, which implies that the remaining conclusions in Theorem I remain valid again (since $T(u)=$ $\left.Y_{0}+V_{\tau(u)}-Y_{\tau(u)}\right)$; cf. Gut (1988), p. 118.

The following result emerges.

Theorem II. The results of Theorem I also hold for the lifetime in the cumulative shock model II.

\section{Remarks}

4.1. If the shocks are non-negative, then the first part of Remark 3.2 also applies to model II; cf. Gut (1988), p. 118.

Note also that in this case $\{\tau(t)-1, t \geqq 0\}$ is the counting process associated with the shocks. This is, however, not true in the general case.

4.2. Sumita and Shanthikumar (1985) also have results for model II.

\section{A remark on insurance risk theory}

In insurance risk theory it is customary to identify the interclaim times with the $W$-sequence and the claims with the $Z$-sequence. Then $V_{\tau(t)-1}$ is the total amount claimed in the time interval $(0, t]$.

If, instead, we identify the claims with the $W$-sequence and the interclaim times with the $Z$-sequence, then $V_{\tau(t)}$ represents the time that has elapsed when the total amount claimed exceeds $t$. Needless to say, this is the cumulative shock model I.

Further, if the initial capital is $z$ and the gross risk premium is $\beta$, then $z+\beta V_{\tau(t)}-U_{\tau(t)}$ is the risk reserve at the time point just described. Moreover, since $U_{\tau(t)} \approx t$ for large $t$ (this can be made precise), $z+\beta V_{\tau(t)}-t$ is an approximation of the risk reserve at this moment. Since, trivially, $U_{\tau(t)}>t$, it follows that this is a (slight) overestimate of the risk reserve.

\section{Acknowledgement}

This paper was written during the author's stay at Instytut Matematyki, UMCS, Lublin, Poland. I wish to thank the Institute, in particular Professors Szynal and Rychlik, for their support, friendship and hospitality. 


\section{References}

Anderson, K. K. (1988) A note on cumulative shock models. J. Appl. Prob. 25, 220-223.

GuT, A. (1988) Stopped Random Walks. Limit Theorems and Applications. Springer-Verlag, New York.

Gut, A. AND JANSON, S. (1983) The limiting behaviour of certain stopped sums and some applications. Scand. J. Statist. 10, 281-292.

Sumita, U. AND Shanthikumar, J. G. (1985) A class of correlated cumulative shock models. Adv. Appl. Prob. 17, 347-366. 